\title{
Development and Validation of a New High-Performance Liquid Chromatography Method for the Simultaneous Quantification of Coenzyme Q10, Phosphatidylserine, and Vitamin C from a Cutting- Edge Liposomal Vehiculization
}

\author{
Marta Ruiz-Garcia, ${ }^{\dagger},, \S$ Pilar Pérez-Lozano, ${ }^{*}, \dagger, \| \odot$ Débora Mercadé-Frutós, ${ }^{\dagger}$ Anna Nardi-Ricart, ${ }^{\dagger}$ \\ Marc Suñé-Pou, ${ }_{\dagger}^{\dagger, \|}$ Mary Cano-Sarabia, ${ }^{\ddagger}$ Sonia Garcia-Jimeno, ${ }^{\ddagger}$ Josep M. Suñé-Negre, ${ }^{\dagger, \|}$ \\ Daniel Maspoch, ${ }^{\ddagger}, \perp$ and Encarna García-Montoya ${ }^{\dagger, \|}$
}

\begin{abstract}
${ }^{\dagger}$ Pharmacy, Pharmaceutical Technology and Physicochemical Department, Faculty of Pharmacy, University of Barcelona, Barcelona 08028, Spain

${ }^{\ddagger}$ Catalan Institute of Nanoscience and Nanotechnology (ICN2), CSIC and The Barcelona Institute of Science and Technology, Bellaterra 08193, Spain

${ }^{\S}$ Vitae Health Innovation S.L., Barcelona 08160, Spain

"Pharmacotheraphy, Pharmacogenetics and Pharmaceutical Technology Research Group, IDIBELL-UB, Bellvitge Hospital, Hospitalet de Llobregat, Barcelona 08908, Spain

${ }^{\perp}$ Institució Catalana de Recerca i Estudis Avançats (ICREA), Barcelona 08010, Spain
\end{abstract}

Supporting Information

\begin{abstract}
A high-performance liquid chromatography (HPLC) method was developed to simultaneously quantify three widely used active substances such as coenzyme Q10, phosphatidylserine, and vitamin C. This new method optimizes current timing and costs in the analyses of these three active substances. Additionally, since the analyzed compounds were encapsulated on a cutting-edge liposomal formulation, further processing was necessary to be developed prior to HPLC analyses. The technique was studied and adequately validated in accordance with the guidelines of the International Council for Harmonisation of Technical Requirements for Pharmaceuticals for Human Use (ICH) regarding selectivity, linearity, accuracy, precision, and robustness. After data treatment of results, linear regressions for all active substances showed an optimal linearity with a correlation coefficient of $>0.999$ in the concentration range between 70 to $130 \%$ of the liposomal formulation and less than a $3 \%$ relative standard deviation (RSD) in accuracy and precision.
\end{abstract}

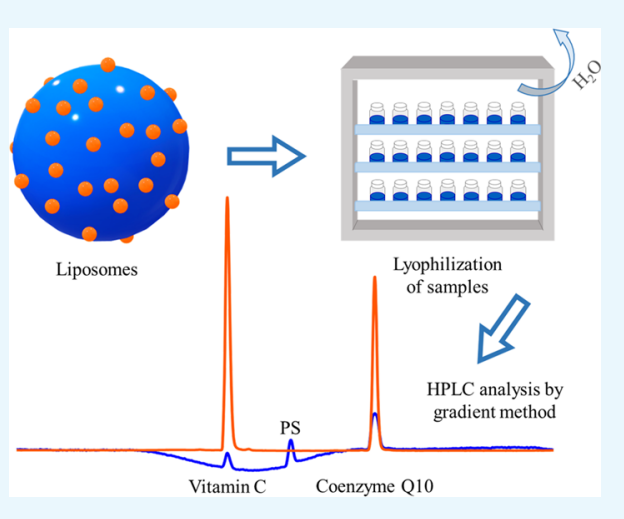

\section{INTRODUCTION}

During the past years, science is accomplishing huge advances in drug discovery through modeling and synthesis toward the treatment of idiopathic diseases. ${ }^{1-4}$ However, even if promising molecules are identified and synthesized, its effectiveness will be reduced or equal to zero if they are unable to achieve the therapeutic target because of a poor absorption. It is estimated that, nowadays, $90 \%$ of the developed molecules on investigation are chemically lipophilic drugs or, in other words, insoluble drugs in water and unable to cross physiological barriers on oral administration.

To enhance drug bioavailability of lipophilic actives, the liposomal technology of encapsulation has been a broadly reported vehicle during the past decades on the literature. Discovered by Bangham and Horne in $1964,{ }^{7}$ this lipid spherical structure allows the insoluble active substances in water to get intertwined with the forming phospholipids of the membrane, while hydrophilic actives can also be encapsulated in its aqueous core. Among many other benefits, this has converted them in an extensively used and very versatile drug delivery technology. ${ }^{8-12}$

Herein, we describe a novel method of analysis for the simultaneous quantification of three coencapsulated active substances with very similar characteristics of hydrophobicity in a liposomal matrix, and also, we explain the pretreatment procedure for these liposomal samples prior to analyses. Specifically, the coanalyzed active substances are the coenzyme Q10 (CoQ10), 6-O-palmitoyl-L-ascorbic acid (the lipid-soluble vitamin C), and phosphatidylserine (PS). These active substances are widely used in both basic research and in the

Received: August 2, 2019

Accepted: October 10, 2019

Published: November 11, 2019 
A)

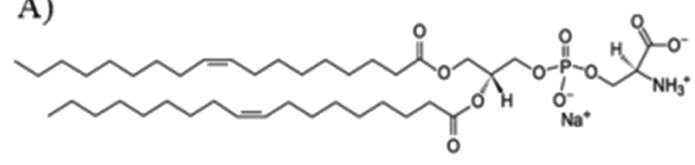

B)

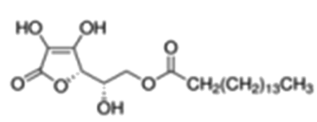

C)

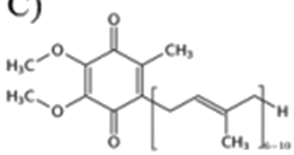

Figure 1. Chemical structure of the analyzed compounds. (A) PS. (B) Vitamin C. (C) CoQ10.

pharmaceutical, cosmetics, and food industry, thus having a high interest and also a huge impact in the economic ambit. In the past 10 years, 5651 articles related with CoQ10 were published in scientific journals, 6969 articles related to PS, and 46,079 articles related with vitamin $C$, all of them with an exponential tendency of increase on each year. ${ }^{13}$ Although many articles have been published describing analytical methodologies of each substance in separate, to the best of our knowledge, there is no published method offering the simultaneous analysis of the three active substances, and even less coencapsulated within the liposomal technology and the additional elements, this means to be considered upon analysis. To the proper determination and quantification of the active substances, it is important to have clear absorption peaks, so we can extrapolate the areas to the actual amount we have of each active substance in the preparation. Since the chemical nature of all the three actives is very similar, it is actually very tricky to elucidate the compounds in separated peaks and avoid them to overlap. Additionally, both coenzyme Q10 and vitamin $\mathrm{C}$ absorb at a wavelength of $275 \mathrm{~nm}$, and all the three substances absorb at $200 \mathrm{~nm}$ wavelength as well, thus making it absolutely necessary to develop a methodology to separate the peaks for its quantification. With combinations of the actives in separate, just one article of simultaneous analysis has been published, reported by Temova-Rakuša et al., ${ }^{14}$ with the coanalysis of coenzyme Q10 and vitamin C. This article provides an interesting method for their quantification but not valid for the analysis of phosphatidylserine. In the Supporting Information (Figure S1), we attach a chromatogram of this reported method with our three active substances, where any absorption peak can be observed for our third active substance, the phosphatidylserine, meaning that it is not a suitable analytical method for this substance or for the simultaneous quantification of the three active substances. Therefore, our method could be considered as a unique and interesting contribution with applications in pharmaceutical/cosmetic/ food industry.

After the design and development of the method, a complete validation study was meticulously accomplished following the ICH quality guidelines. It was also used and proved in different real circumstances such as long-term stability studies at different conditions.

\section{RESULTS AND DISCUSSION}

Sample Preparation Strategy. After some assays, results demonstrated that it is necessary to remove the water from the samples to allow the elution of substances at the chromatogram and to successfully analyze and quantify the peaks of the active substances. First, with any water in the sample, it was almost impossible to correctly solve all the lipids. Second, being hydrophobic active substances with water, the peaks were not eluting in a satisfactory peak form or were not eluting at all. Freeze-drying of samples was carried out. Afterwards, twenty different lyophilized vials with the liposomal formulation were injected and analyzed with the developed HPLC method to test the accuracy of the sample preparation strategy, and the RSD after the analyses of the all the vials was $2.35 \%$ for CoQ10, $2.14 \%$ for vitamin C, and $2.74 \%$ for PS, thus showing optimal results of repeatability.

Method Development. The designed HPLC method was meant to properly analyze the content of CoQ10, vitamin C, and PS from a liposomal formulation. First, we assayed different mixtures and proportions between methanol, isopropanol, acetonitrile, and water buffer. To choose an adequate buffer, we looked out to the $\mathrm{p} K_{\mathrm{a}}$ of the three substances and selected buffer at a $\mathrm{pH}$ where all compounds would be in an undissociated form, such as $0.02 \mathrm{M} \mathrm{NH}_{4} \mathrm{AcO}$ at $\mathrm{pH}=6.5$. Figure 1 shows the molecular structure of every active substance, while its corresponding $\mathrm{p} K_{\mathrm{a}}$ partition coefficient, and molecular weight are shown in Table 1 . The

Table 1. Chemical Properties To Be Considered for the Design of the Method ${ }^{15-17}$

\begin{tabular}{llll} 
parameter & \multicolumn{1}{c}{ PS } & vitamin C & CoQ10 \\
$\log P$ & 10.55 & 6 & 10 \\
$\mathrm{MW}(\mathrm{g} / \mathrm{mol})$ & 810.025 & 414.539 & 863.365 \\
$\mathrm{p} K_{\mathrm{a}}$ & $9.38 ; 1.47$ & $4.36 ;-3$ & -4.7 \\
\hline
\end{tabular}

first chromatographic meticulously tested conditions showed us that for a favorable elution of CoQ10, a 100\% flow of pure methanol or isopropanol is needed, and any mixture with water made its elution impossible with an optimal peak shape. On the other hand, for the analysis of vitamin C, a mixture of organic solvent and water was needed for its proper elution, and PS was not eluted in any isocratic condition with an optimal peak shape, considering theoretical plate numbers $(N)$ and USP tailing factor.

At this point of development, a gradient of $\mathrm{NH}_{4} \mathrm{AcO}$ buffer with isopropanol was extensively investigated at different proportions and with different gradient duration times until we found an adequate combination that allowed the optimal elution of all the compounds during the same analysis. An example of the obtained chromatogram after last optimization is shown in Figure 2.

Method Validation. Before carrying through the validation assays, the suitability of the equipment with the method was tested. Table 2 shows RSD of retention times (RT), symmetry of the peak, USP tailing factor, capacity factor, and $N$, demonstrating consistency and appropriateness. The tabulated retention times of the six analyzed samples and symmetry analyses on each peak of the active substances can be found in the Supporting Information (Scheme S2 and Figures S3-S5).

After checking the suitability of the equipment and before starting any other validation step, it was necessary to understand for how long samples are stable above at least $95 \%$ of all active substances. It was determined that, to accomplish this requirement, liposomal samples could be injected up to $3 \mathrm{~h}$ after its preparation in the HPLC vials without significant degradation of any sample, while for the standard solution, the time was shortened to $1 \mathrm{~h}$. So, it is 


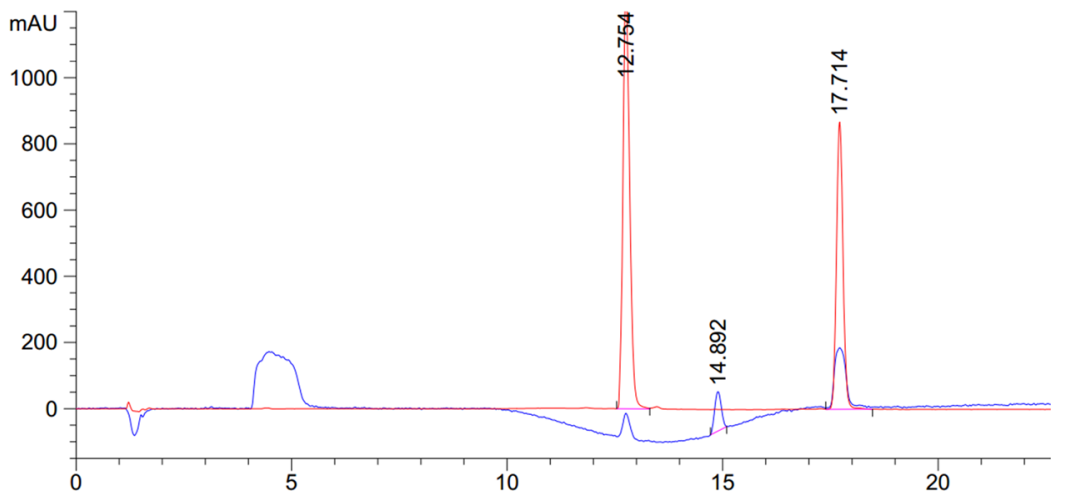

Figure 2. Chromatographic exemplification of the developed method with the three analyzed active substances. The peak with an RT of 12.754 min corresponds to vitamin C, an RT of 14.892 min corresponds to PS, and an RT of 17.714 corresponds to CoQ10. The red line is $\lambda=275 \mathrm{~nm}$, while the blue line is $\lambda=200 \mathrm{~nm}$.

Table 2. Suitability of the Equipment and Data Analyses

\begin{tabular}{llll}
\multicolumn{1}{c}{ parameter } & \multicolumn{1}{c}{ PS } & vitamin C & CoQ10 \\
retention times $(\mathrm{min})$ & 14.86 & 12.72 & 17.73 \\
retention times (RSD \%) & 0.09 & 0.25 & 0.14 \\
symmetry of peak & 1.01 & 0.73 & 0.89 \\
USP tailing factor & 1.22 & 1.23 & 1.08 \\
capacity factor & 3.08 & 2.49 & 3.85 \\
plate numbers $(N)$ & 40,891 & 33,327 & 67,904
\end{tabular}

highly recommended to analyze samples right after its solubilization in organic solvents and preparation for HPLC quantification.

The precision of the system was also checked before any other validation step. After five consecutive injections of a $100 \%$ concentration of PS, the area scattered only in a $0.59 \%$, being inside our acceptance criteria of $\leq 1.0 \%$, thus confirming its reliability (see the Supporting Information).

Selectivity was confirmed by overlapping the chromatograms of an analyzed sample with a standard solution and a third one with the rest of the substances (Figure 3). Additionally, the purity factor of peaks was studied. In all cases, purity was above the threshold limit, set at 990.000 (see the Supporting Information, Table S6 and FiguresS7 and S8).

Through analyzing all seven concentrations between 130 and $70 \%$ of the standard solution, results demonstrated an adequate linearity in this concentration range. Data is summarized on Table 3, with RSD and correlation coefficients accomplishing our established limits. Table 3 also shows data regarding precision and accuracy.

For the precision of the method evaluated in different days, we established a $2.8 \%$ RSD and a $3.7 \%$ for repeatability (intralaboratory). Regarding accuracy, every recovery percentage after the study was included in our quality specifications. All this data demonstrated excellent results with respect to the validation of the method and indicated that this method is precise and accurate.

Table 4 details the percentage of recovery on the nine analyses at different concentrations for evaluating accuracy. Every concentration resulted in less than a $2 \%$ RSD, and ANOVA test showed nonsignificant differences on any active substance at any concentration.

Lastly, robustness studies revealed that the method must be controlled in terms of temperature, injection volume, wavelength detection, and flow since otherwise results could slightly variate. Being a method with three analyzed compounds, each
A)

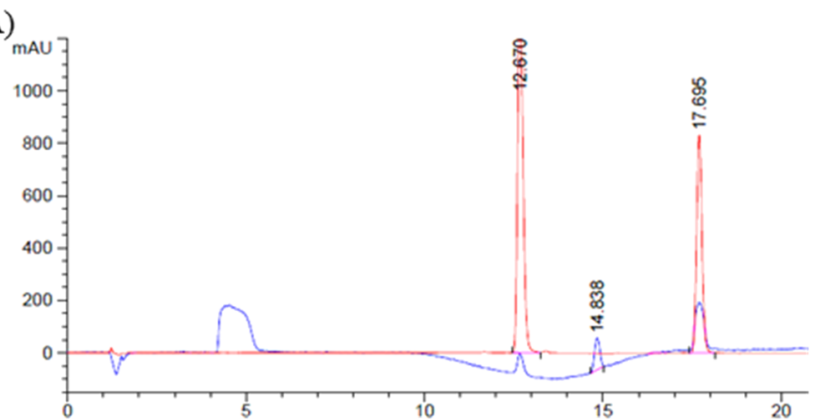

B)

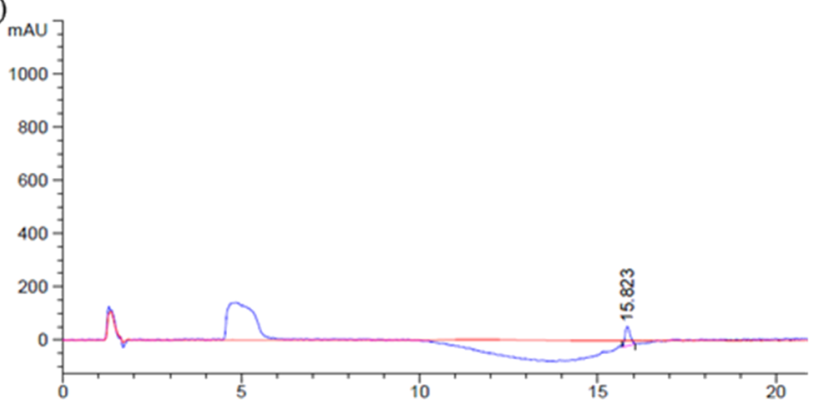

C)

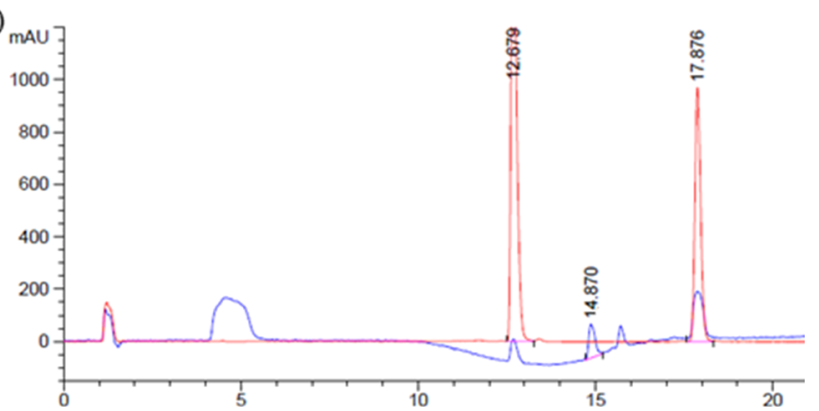

Figure 3. Representative chromatograms. (A) Standard solution. (B) Mixture of the other lipids: PC, Chol, and Chol-PEG. (C) Liposomal sample.

parameter resulted in some degree of retention time differences or peak areas.

Table 5 indicates which parameter showed significant or nonsignificant differences after deliberated changes upon its analysis with a one-way ANOVA test. On this table, although significant differences were shown in many of the deliberated changes, RSD values were not in any case over $5 \%$ but with wavelength for PS. Since $\lambda=200 \mathrm{~nm}$ is a wavelength where also solvents have certain absorbance, it is a critical parameter 
Table 3. Summarized Data of Linearity, Precision, and Accuracy during the Validation of the Method

\begin{tabular}{|c|c|c|c|c|c|c|c|}
\hline \multirow[b]{2}{*}{ analytes } & \multicolumn{3}{|c|}{ linearity } & \multicolumn{3}{|c|}{ precision } & \multirow{2}{*}{$\begin{array}{c}\text { accuracy } \\
\text { recovery }(\%, \max \text { and } \min \\
\text { values })\end{array}$} \\
\hline & $\begin{array}{c}\text { range } \\
(\mathrm{mg} / \mathrm{mL})\end{array}$ & $\begin{array}{l}\text { correlation } \\
\text { coefficient }\end{array}$ & $\begin{array}{l}\text { RF RSD } \\
(\%)\end{array}$ & $\begin{array}{l}\text { standard solution, } \\
\text { RSD (\%) }\end{array}$ & $\begin{array}{c}\text { different days, RSD } \\
(\%)\end{array}$ & $\begin{array}{l}\text { different analysts, } \\
\text { RSD (\%) }\end{array}$ & \\
\hline PS & $1.008-0.543$ & 0.999 & 1.82 & 1.97 & 2.80 & 3.18 & $101.35-98.25$ \\
\hline CoQ10 & $0.312-0.168$ & 0.999 & 1.01 & 0.97 & 2.45 & 1.89 & $101.92-98.30$ \\
\hline vitamin $C$ & $0.260-0.140$ & 1.000 & 0.89 & 1.96 & 2.80 & 1.89 & $101.89-98.28$ \\
\hline
\end{tabular}

Table 4. Detailed Results of Studying the Accuracy

\begin{tabular}{llll}
\multicolumn{1}{c}{ parameter } & \multicolumn{1}{c}{ PS } & \multicolumn{1}{c}{ vitamin C } & \multicolumn{1}{c}{ CoQ10 } \\
$\begin{array}{l}\text { recovery at } 130 \% \\
(\%)\end{array}$ & $100.21 \pm 1.61$ & $101.20 \pm 0.93$ & $100.21 \pm 1.82$ \\
$\begin{array}{l}\text { recovery at } 100 \% \\
(\%)\end{array}$ & $98.42 \pm 0.23$ & $100.28 \pm 1.83$ & $100.17 \pm 1.01$ \\
$\begin{array}{l}\text { recovery at } 70 \%(\%) \\
\text { ANOVA test } \\
(p \text {-value })\end{array}$ & $98.48 \pm 1.26$ & $100.98 \pm 0.80$ & $99.71 \pm 0.93$ \\
& 0.98 & 0.67 & 0.88
\end{tabular}

Table 5. Summarized Statistics from ANOVA Test on Robustness $^{a}$

\begin{tabular}{llllllll} 
& \multicolumn{2}{c}{ PS } & \multicolumn{2}{c}{ vitamin C } & \multicolumn{2}{c}{ CoQ10 } \\
parameter & $p$-value & $\begin{array}{c}\text { RSD } \\
(\%)\end{array}$ & $p$-value & $\begin{array}{c}\text { RSD } \\
(\%)\end{array}$ & $p$-value & $\begin{array}{c}\text { RSD } \\
(\%)\end{array}$ \\
$\begin{array}{l}\text { temperature } \\
\text { injection }\end{array}$ & 0.239 & 1.72 & $>0.01 *$ & 4.47 & $>0.01 *$ & 1.35 \\
$\quad$ volume & 0.225 & 3.34 & 0.792 & 1.15 & $0.032^{*}$ & 0.70 \\
wavelength & $>0.01 *$ & 14.88 & $0.013^{*}$ & 1.85 & 0.135 & 0.75 \\
flow & 0.695 & 2.17 & $>0.01 *$ & 7.22 & 0.341 & 0.92 \\
$a *$ ind & & & &
\end{tabular}

$a_{*}$ indicates that there are significant statistical differences.

to be controlled. Specifically, a two-tailed T-student test was carried out to compare wavelengths $197 \mathrm{~nm}$ versus $200 \mathrm{~nm}$ and $200 \mathrm{~nm}$ versus $203 \mathrm{~nm}$. The first T-test contained highly significant differences with a $p$-value of $>0.01$, but nonsignificant differences were observed with a $p$-value of 0.80 between 200 and $203 \mathrm{~nm}$, thus being $203 \mathrm{~nm}$ also a suitable wavelength to be used.

Based on the standard deviation of the response and the slope, based on the calibration curve, LOD and LOQ were calculated for three analytes. Results are presented in Table 6.

Table 6. LOD and LOQ Results

\begin{tabular}{cccc} 
parameter & PS & vitamin C & CoQ10 \\
LOD $(\mathrm{mg} / \mathrm{mL})$ & 0.082 & 0.018 & 0.021 \\
LOQ $(\mathrm{mg} / \mathrm{mL})$ & 0.249 & 0.054 & 0.063 \\
\hline
\end{tabular}

Applications of the Method. Once validated, the proposed method was used to accurately evaluate the stability of the three active substances encapsulated on the mentioned liposomal formula after 3 months of storage at 4 and $25{ }^{\circ} \mathrm{C}$. This study verifies the applicability of the method in a real case study. Results are presented in Table 7 , while an illustrative chromatogram of each temperature is included in the Supporting Information (Figures S9 and S10).

\section{CONCLUSIONS}

The developed method demonstrated promising results on the simultaneous analyses of CoQ10, vitamin C, and PS. To the best of our knowledge, this is the first reported method for the determination of these widely used active substances at the same time. Thus, this method could result in an ideal analytic
Table 7. Results of Stability Studies with the Developed Method after 3 Months

$\begin{array}{cccc}\text { temperature condition } & \text { PS } & \text { vitamin C } & \text { CoQ10 } \\ 4{ }^{\circ} \mathrm{C}(\%) & 100.09 \pm 0.31 & 95.27 \pm 0.58 & 97.56 \pm 0.20 \\ 25{ }^{\circ} \mathrm{C}(\%) & 96.19 \pm 0.22 & 85.57 \pm 0.15 & 87.27 \pm 0.23\end{array}$

method for the cosmetics, pharmaceutical, and food supplement industry.

Additionally, the formulation involving the liposomal technology may be a tricky challenge to allow a correct quantification and analyses, especially for very lipophilic active substances to which the presence of water can signify a complicated limitation for the analyses. The performed methodology of sample treatment prior to its analyses may be as well an additional step of valuable interest. The concept may be applied to any other possible liposomal formulations with highly hydrophobic active substances.

In terms of validation of the method, following the $\mathrm{ICH}$ guidelines, the suitability of the equipment, selectivity of the method, linearity, robustness, precision, accuracy, and stability of the solution during the analyses were tested with positive and encouraging results.

Lastly, with the purpose of verifying the applicability of the method, a testing of the method with a real case was carried out. Samples subjected to stability studies were analyzed, and results were studied and interpreted, with excellent results.

\section{EXPERIMENTAL SECTION}

Materials and Chemical Reagents. 6-O-Palmitoyl-Lascorbic acid (vitamin $\mathrm{C}$; analytical standard, $\geq 99.0 \%$ ), cholesterol-(polyethylene glycol-600) (Chol-PEG), and cholesterol (Chol) were obtained from Sigma-Aldrich (Darmstadt, Germany). 1,2-Dioleoyl-sn-glycero-3-phospho-L-serine sodium salt (phosphatidylserine or PS; analytical standard, $\geq 99.0 \%$ ) and 1,2-dimyristoyl-sn-glycero-3-phosphocholine (phosphatidylcholine or PC) were purchased from Lipoid $\mathrm{GmbH}$ (Switzerland). Coenzyme Q10 (CoQ10; United States Pharmacopeia (USP) reference standard quality, $\geq 99.0 \%$ ) was obtained from Vitae Health Innovation S.L (Spain). Ammonium acetate $\left(\mathrm{NH}_{4} \mathrm{AcO}\right)$ was acquired from Panreac Quimica (Barcelona, Spain).

Instrumentation. Analyses were carried out with an HPLC system model $1100 \mathrm{HP}$ from Agilent (Agilent Technologies, Santa Clara, EEUU), composed of a quaternary pump, an automatic injector, a diode array detector, and a column oven where our column was placed, all controlled by a ChemStation software version B04.02. For the freeze-drying of samples, a Telstar Cryodos-50 lyophilizator was used.

Preparation of Liposomes. Liposomal formulations were prepared using the thin-film hydration method with some modifications. ${ }^{18}$ Briefly, PC, PS, Chol, Chol-PEG, and CoQ10 were dissolved in chloroform solutions $(100 \mathrm{mg} / \mathrm{mL})$ or chloroform/ethanol 7:3 for vitamin $\mathrm{C}$ and mixed afterward at 
the desired molar. In this case, the molar concentration was 79 $\mathrm{mM}$, with $30.4 \%$ PS, $22.8 \%$ PC, $19.0 \%$ Chol, $15.3 \%$ vitamin C, $8.8 \%$ CoQ10, and 3.8\% Chol-PEG. The organic solvent was removed under vacuum and nitrogen to afford a dry lipid film, which was hydrated with an aqueous solution under vigorous stirring for $15 \mathrm{~min}$ at $40{ }^{\circ} \mathrm{C}$. Under these conditions, the stacks of liquid crystalline lipid bilayers become fluid and swell, resulting in their detachment during agitation and their selfclosure to form multilamellar large vesicles (MLVs). Small unilamellar vesicles (SUVs) were obtained by homogenizing the MLV suspension using an extruder (Lipex Biomembranes, Canada) and a polycarbonate membrane (pore size, $200 \mathrm{~nm}$ ).

Sample Preparation Strategy. The treatment and preparation of samples prior to HPLC analyses were essential for allowing the quantification of the lipidic active substances. Liposomal formulations have by definition the characteristic of including both water and water-insoluble compounds, and this could make any posterior analyses difficult. Since the presence of water is a very unfavorable condition to dissolve and allow the flow of the substances through the column in an appropriate way, a procedure of analysis including the lyophilization of the samples was carried out: an scheme illustrating the preparation process of the samples can be found in the Supporting Information (Scheme S2). For the lyophilization of samples, $1 \mathrm{~mL}$ of the liposomal solution was pipetted on each vial and frozen afterward at $-80{ }^{\circ} \mathrm{C}$ on an ultralow temperature freezer for $12 \mathrm{~h}$. Once samples were completely frozen, they were freeze-dried for 4 days. Lyophilization conditions of pressure and temperature were $1 \mathrm{mbar}$ and $-50{ }^{\circ} \mathrm{C}$, respectively.

Once samples were completely dried, $2 \mathrm{~mL}$ of $\mathrm{CHCl}_{3}$ was added to properly solubilize the very apolar active substances: PS, vitamin C, and CoQ10. After 5 min of slight shaking and only when the sample was completely dissolved, $10 \mathrm{~mL}$ of methanol was added, and the final solution was transferred into $25 \mathrm{~mL}$ volumetric flasks. The vials were afterward cleaned with methanol, which was also transferred to the flasks, making up to $25 \mathrm{~mL}$ with more methanol. Lastly, all solutions were filtered with $0.45 \mu \mathrm{m}$ PVDF filters, and HPLC vials were prepared. The final concentration of each active compound at the moment of injection to the HPLC system was $776 \mu \mathrm{g}$ $\mathrm{mL}^{-1} \mathrm{PS}, 240 \mu \mathrm{g} \mathrm{mL}^{-1} \mathrm{CoQ} 10$, and $200 \mu \mathrm{g} \mathrm{mL}^{-1}$ vitamin C.

To validate the preparation procedure developed, 20 lyophilized vials were injected, establishing an RSD limit of $2.7 \%$.

Standard Solution Preparation. For the preparation of a standard solution, $19.4 \mathrm{mg}$ of PS, $6 \mathrm{mg}$ of CoQ10, and $5 \mathrm{mg}$ of vitamin $\mathrm{C}$ were accurately weighed and dissolved in the same way than in the sample preparation procedure, so the final peak areas would be also the same both in standard solutions and sample solutions.

Chromatographic Conditions. For HPLC analyses, the employed column was a C18 Zorbax Eclipse Plus $2.1 \times 150$ $\mathrm{mm}(5 \mu \mathrm{m})$, with a constant flow of $0.25 \mathrm{~mL} \mathrm{~min}{ }^{-1}$ and a controlled temperature of $25{ }^{\circ} \mathrm{C}$. The injection volume was 10 $\mu \mathrm{L}$, and the detection wavelengths were $\lambda=275 \mathrm{~nm}$ for vitamin $\mathrm{C}$ and $\mathrm{CoQ} 10$ and $\lambda=200 \mathrm{~nm}$ for PS since no other absorbance was found after checking with the diode array accessory. After $19 \mathrm{~min}$, all the compounds were already eluted, but the stop time was fixed at $28 \mathrm{~min}$ to return to the initial conditions and stabilize the equipment. The detailed proportions of the two employed solvents during the time are shown in Table 8.
Table 8. Detailed Gradient of the Developed Method

time $(\mathrm{min})$
0
5
10
18
19
28

solvent B (\%)
60
60
0
0
60
60
solvent A (\%)

A gradient method was developed and is detailed in Table 8 . Solvent A in the table is isopropanol, while Solvent B is a buffer solution of $0.02 \mathrm{M} \mathrm{NH}_{4} \mathrm{AcO}$.

Method Validation. The development of the method was followed by validation studies to ensure selectivity, linearity, robustness, precision, stability of the solution during the analyses, and accuracy of the method, following the ICH guidelines. ${ }^{19}$ Prior to any validation test, six injections of a standard solution were analyzed to ensure the suitability of the equipment. Retention times with less than $1.0 \%$ variations, capacity numbers greater than 2, and symmetry of peaks between 0.7 and 1.2, theoretical plate numbers $(N)$ above 2000 for any peak, and USP tailing factor smaller than 1.5 were checked out.

Selectivity is the ability to assess unequivocally the analyte in the presence of other components, which may be expected to be present. For this study, both sample and standard solutions were compared, thus confirming the complete separation of all compounds with a purity peak test being carried out for each active substance. Linearity was tested by preparing seven different concentrations per triplicate between 70 and $30 \%$ of the standard solution at the usual working concentration, studying if the response is directly proportional within this range. Response factor (RF) was the analyzed parameter on linearity, being the result of dividing peak areas by the corresponding concentration. Precision is the parameter that expresses the degree of scatter between a series of measures. In this case, the precision of the method and system was evaluated with the injection of nine standard solutions at different concentrations and with different analysts and different days of work, studying its repeatability. Robustness is a measure of the capacity to remain unaffected by small but deliberated variations in method parameters such as the temperature of the column $\left( \pm 3{ }^{\circ} \mathrm{C}\right)$, the analyzed wavelength $( \pm 3 \mathrm{~nm})$, the injection volume $( \pm 5 \mu \mathrm{L})$, or modifications in the flow level of the pump $( \pm 0.05 \mathrm{~mL} / \mathrm{min})$. This study is necessary to understand the importance of controlling a determinate parameter. Stability of the active substances in the HPLC vials once prepared is also vital to understand for how long the solution is stable before injecting. Lastly, the accuracy of the method was assessed to verify if any of the lipids of the liposome affect the analyses of the active substances. To this end, three standard solutions and the other lipids were prepared at 130,100 , and $70 \%$ of the usual working concentration, and real concentrations were compared with the theoretical ones. According to the American Association of Official Analytical Chemists $\left(\mathrm{AOAC}^{20}\right)$, the limits for the RSD (coefficient of variation) are established in consonance with the concentration of the analyte in the sample, so it established a limit for the active substances of $2.7 \%$ RSD. 


\section{ASSOCIATED CONTENT}

\section{S Supporting Information}

The Supporting Information is available free of charge on the ACS Publications website at DOI: 10.1021/acsomega.9b02456.

Instrumentation details, statistical parameters explained in the text, graphical chromatograms from validation, and graphical examples of real analyses (PDF).

\section{AUTHOR INFORMATION}

\section{Corresponding Author}

*E-mail :perezlo@ub.edu.

\section{ORCID}

Pilar Pérez-Lozano: 0000-0001-6899-066X

Daniel Maspoch: 0000-0003-1325-9161

\section{Author Contributions}

The manuscript was written through contributions of all authors. All authors have given approval to the final version of the manuscript.

\section{Funding}

This work was supported by Industrial Doctorates Grant [grant number DI55, 2016], as well as the support to ICN2 by the Severo Ochoa program of the Spanish Ministry of Economy, Industry and Competitiveness (MINECO, grant no. SEV-2017-0706), and the funding by the CERCA program/Generalitat de Catalunya.

\section{Notes}

The authors declare no competing financial interest.

\section{ACKNOWLEDGMENTS}

The authors want to render thanks to the Analytical Department of Chemistry at the University of Barcelona to its advice during the development of the analytical method.

\section{REFERENCES}

(1) Ganesan, A.; Coote, M. L.; Barakat, K. Molecular dynamicsdriven drug discovery: leaping forward with confidence. Drug Discovery Today 2017, 22, 249-269.

(2) Chen, H.; Engkvist, O.; Wang, Y.; Olivecrona, M.; Blaschke, T. The rise of deep learning in drug discovery. Drug Discovery Today 2018, 23, 1241-1250.

(3) De Vivo, M.; Masetti, M.; Bottegoni, G.; Cavalli, A. Role of Molecular Dynamics and Related Methods in Drug Discovery. J. Med. Chem. 2016, 59, 4035-4061.

(4) Taylor, A. P.; Robinson, R. P.; Fobian, Y. M.; Blakemore, D. C.; Jones, L. H.; Fadeyi, O. Modern advances in heterocyclic chemistry in drug discovery. Org. Biomol. Chem. 2016, 14, 6611-6637.

(5) Benet, L. Z.; Broccatelli, F.; Oprea, T. I. BDDCS Applied to Over 900 Drugs. AAPS J. 2011, 13, 519-547.

(6) Wang, G.; Wang, J.; Wu, W.; To, S. S. T.; Zhao, H.; Wang, J. Advances in lipid-based drug delivery: enhancing efficiency for hydrophobic drugs. Expert Opin. Drug Delivery. 2015, 12, 1475-1499.

(7) Bangham, A. D.; Horne, R. W. Negative staining of phospholipids and their structural modification by surface-active agents as observed in the electron microscope. J. Mol. Biol. 1964, 8, 660-IN10.

(8) Wagner, A.; Vorauer-Uhl, K. Liposome Technology for Industrial Purposes. J. Drug Delivery. 2011, 591325.

(9) Khorasani, S.; Danaei, M.; Mozafari, M. R. Nanoliposome technology for the food and nutraceutical industries. Trends Food Sci. Technol. 2018, 79, 106-115.
(10) Kaul, S.; Gulati, N.; Verma, D.; Mukherjee, S.; Nagaich, U. Role of Nanotechnology in Cosmeceuticals: A Review of Recent Advances. J. Pharm 2018, 1-19.

(11) Torchilin, V. P. Recent advances with liposomes as pharmaceutical carriers. Nat. Rev. Drug Discovery 2005, 4, 145-160.

(12) Pattni, B. S.; Chupin, V. V.; Torchilin, V. P. New Developments in Liposomal Drug Delivery. Chem. Rev. 2015, 115, 10938-10966.

(13) Results of coenzyme Q10 plus ubiquinone, vitamin $c$ and phosphatidylserine, respectively; https://apps-webofknowledge-com. sire.ub.edu/UA GeneralSearch input.do?product=UA\&search $\mathrm{mode}=\mathrm{G}$ e n e ral S e a r c h \& S I D =

F211r8c4dns9yyfCoSK\&preferencesSaved (accessed Feb 12, 2019)

(14) Temova-Rakuša, Z.; Srečnik, E.; Roškar, R. Novel HPLC-UV Method for Simultaneous Determination of Fat-soluble Vitamins and Coenzyme Q10 in Medicines and Supplements. Acta Chim. Slov. 2017, 64, 523-529.

(15) Drug Bank bioinformatics and cheminformatics data base; https://www.drugbank.ca/drugs/. (accessed March 26, 2019)

(16) PubChem chemistry data base; https://pubchem.ncbi.nlm.nih. gov/. (accessed March 26, 2019)

(17) ChemAxon Ltd. Calculators and Predictors; https://chemaxon. com/. (accessed July 8,2019 )

(18) Szoka, F., Jr.; Papahadjopoulos, D. Comparative Properties and Methods of Preparation of Lipid Vesicles (Liposomes). Annu. Rev. Biophys. Bioeng. 1980, 9, 467-508.

(19) International conference on harmonisation of technical requirements for registration of pharmaceuticals for human use. ICH harmonised tripartite guideline. VALIDATION OF ANALYTICAL PROCEDURES: TEXT AND METHODOLOGY Q2 (R1). 2005. https:// ich.org/ (accessed March 26, 2019)

(20) AOAC Guidelines for Single Laboratory Validation of Chemical Methods for Dietary Supplements and Botanicals. (n.d.). www.AOAC. org. (accessed July 8, 2019) 